\title{
Management of Foley catheter induction among nulliparous women: a retrospective study
}

Heidi Kruit ${ }^{1 *}$, Oskari Heikinheimo ${ }^{1}$, Veli-Matti Ulander ${ }^{1}$, Ansa Aitokallio-Tallberg ${ }^{1}$, Irmeli Nupponen², Jorma Paavonen ${ }^{1}$ and Leena Rahkonen ${ }^{1}$

\begin{abstract}
Background: Induction of labour is associated with increased risk for caesarean delivery among nulliparous women. The aims of this study were to evaluate the risk factors for caesarean delivery and to investigate the risk of maternal and neonatal infections in nulliparous women undergoing induction of labour by Foley catheter.

Methods: This clinical retrospective study of 432 nulliparous women with singleton pregnancy and intact amniotic membranes at or beyond 37 gestational weeks scheduled for induction of labour by Foley catheter was conducted over the course of one year, between January 2012 and January 2013, in Helsinki University Hospital. The main outcome measures were caesarean section rate and maternal and neonatal infections. Univariate and multivariate logistic regressions were used to estimate relative risks by odds ratios with $95 \%$ confidence intervals.

Results: The caesarean section rate was $39.1 \%(n=169)$. In multivariate regression analysis, the factors associated with caesarean section were the need for oxytocin for labour induction [OR $2.9(95 \% \mathrm{Cl} 1.8-4.5) p<0.001$ ] and early epidural analgesia [OR 9.9 (95\% Cl 2.1-47.5), $p=0.004]$. The maternal intrapartum infection rate was $6.3 \%$, and the clinical neonatal infection rate was $2.8 \%$. In multivariate analysis, gestational diabetes was associated with maternal intrapartum infection [OR 4.3 (95\% Cl 1.7-11.0, $p=0.002$ ] and early epidural analgesia with neonatal clinical sepsis [OR $10.5(95 \% \mathrm{Cl} 1.4-76), p=0.02]$.

Conclusions: Oxytocin induction and early epidural analgesia were associated with caesarean delivery. Gestational diabetes and early epidural analgesia were associated with infectious morbidity. Since the first caesarean delivery has a major impact on subsequent pregnancies, optimising labour induction among nulliparous women is important.
\end{abstract}

Keywords: Induction of labour, Foley catheter, Caesarean delivery rate, Nulliparous, Maternal infection, Neonatal infection

\section{Background}

The rates of induction of labour (IOL) are rising, ranging between 20-30\% in developed countries [1, 2]. Both mechanical and pharmacological methods are used for IOL in women with an unfavourable cervix, and vaginal delivery rates are similar [3]. WHO recommends the use of a balloon catheter for IOL (WHO Recommendations for Induction of Labour, World Health Organization,

\footnotetext{
*Correspondence: heidi.kruit@hus.fi

'Department of Obstetrics and Gynecology, University of Helsinki, Helsinki University Hospital, Haartmaninkatu 2, Helsinki 00029 HUS, Finland Full list of author information is available at the end of the article
}

2011). However, both IOL methods are associated with induction failure and caesarean delivery $[4,5]$. The risk of caesarean section in such cases is high, up to $50 \%$, especially among nulliparous women [6-8]. We found earlier that nulliparous women with prolonged pregnancy and Foley catheter IOL in our hospital had a high caesarean delivery rate of $37.3 \%$ [9]. The reasons for increased caesarean section rates are unclear, but they may in part relate to the subsequent management of induced labour. In addition, previous studies have shown that duration of labour correlates with the risk of maternal chorionamnionitis and neonatal infections $[9,10]$. This 
highlights the need for additional studies. Thus, we wanted to further analyse the factors that may affect the progress of induced labour. The primary objective of the study was to describe labour outcomes in nulliparous women undergoing IOL by Foley catheter, while the secondary objective was to evaluate risk factors for caesarean delivery and maternal and neonatal infections.

\section{Methods}

This clinical retrospective study of 532 nulliparous women with singleton pregnancy $\geq 37$ weeks of gestation undergoing IOL by Foley catheter was conducted in the Department of Obstetrics and Gynaecology at the Helsinki University Central Hospital between January 2012 and January 2013. The study protocol was approved by the Helsinki and Uusimaa Hospital District ethics committee for obstetrics and gynaecology, pediatrics and psychiatry (Nr. 268/13/03/03/2012). Approval to carry out the study was granted by the Hospital district of Helsinki and Uusimaa (\$20/12042012). The need to collect an informed consent of the participating women was deemed unnecessary by the ethics committee based on national regulations (Medical Research Act 488/1999, chapter 2 a (23.4.2004/ 295), section 5 and 10a). The patients were identified from hospital records. Exclusion criteria were twin pregnancy $(n=7)$, breech presentation $(n=11)$, prelabour rupture of membranes $(n=23)$ and sequential use of Foley catheter and prostaglandin for IOL $(n=59)$, leaving a total of 432 women. All women had a Bishop score $<6$ at the start of IOL.

A single balloon catheter (Rüsch 2-way Foley, Couvelaire tip, catheter size $22 \mathrm{Ch}$, Teleflex Medical, Athlone, Ireland) was used. The catheter was introduced into the endocervix and the space between the amniotic membrane and the lower uterine segment. The balloon reservoir was inflated with a median of $40 \mathrm{ml}$ (range 30-60 ml) of saline. Thereafter, the balloon was pulled so that it rested on the internal os. Light traction was applied, and the catheter was taped to the inner thigh.

After expulsion of the balloon, amniotomy was performed if the Bishop score was $\geq 6$. If spontaneous expulsion of the Foley catheter did not occur within 24 hours, the balloon was removed. If the cervix remained unripe with Bishop score $<6$ after balloon expulsion or removal, induction was continued with intravaginal misoprostol. These $(n=59)$ women were excluded from the final analysis.

Interval times were calculated from the hospital records. The duration of Foley catheter retention was defined as the interval time from balloon insertion to expulsion or removal. The timing of amniotomy varied from immediately after expulsion of the balloon to the next morning, depending on cervical ripeness and delivery unit capacity. Amniotomy was categorised as either immediate ( $\leq 2 \mathrm{~h}$ from balloon expulsion) or delayed (>12 $\mathrm{h}$ from balloon expulsion).

Oxytocin for labour induction was administered in the absence of spontaneous regular contractions. Oxytocin was initiated by discretion of the obstetrician; the timing of its administration varied between 2 and 24 hours after spontaneous rupture of membranes. Oxytocin was also routinely used for labour augmentation if needed, but this study only concentrated on the timing of oxytocin for labour induction. Oxytocin induction was categorised as either immediate ( $\leq 3 \mathrm{~h}$ from rupture of membranes) or delayed ( $>12 \mathrm{~h}$ from rupture of membranes). Regular contractions were defined as contractions every 3 to 5 minutes with cervical dilation of minimum of $3 \mathrm{~cm}$. The induction-to-delivery interval was defined as the time from insertion of the balloon catheter to delivery.

Data on the study population characteristics, maternal antenatal risk factors, mode of delivery, and maternal and neonatal infections were obtained and collected from antenatal clinic charts and hospital records. The main outcomes were the mode of delivery and maternal and neonatal infections. When there was more than one indication for caesarean section, the primary indications were categorised using the following hierarchy: foetal distress, infection, failure to progress and failed induction. Labour arrest in the first stage of labour was defined as failure to progress despite ruptured membranes and a minimum of 4 hours of adequate uterine activity without cervical change. Failed induction was diagnosed after ruptured membranes and 6-12 hours of oxytocin administration without cervical change [11].

Maternal infections were categorised as intrapartum or postpartum (from delivery to discharge). The criteria for intrapartum infection were maternal fever $\left(\geq 38^{\circ} \mathrm{C}\right)$ during labour, foetal tachycardia ( $\geq 160 \mathrm{bpm}$ ), uterine tenderness, purulent amniotic fluid or vaginal discharge and total white cell count $>20$ e9/l. At least two of these criteria had to be met in combination with administration of antibiotics. Postpartum infections included endometritis, wound infection and puerperal fever of unknown origin.

Neonatal infections were categorised into blood culturepositive sepsis, clinical sepsis, and suspected sepsis. Neonatal clinical sepsis was defined as a blood culturenegative infection with symptoms and signs consistent with sepsis (such as respiratory distress, apnea, tachycardia, poor perfusion, low blood pressure, fever, hypo- or hyperglycemia, irritability, feeding problems, lethargy and convulsions), abnormal blood values (such as elevated levels of C-reactive protein, leucocytosis or leucopenia, increased neutrophil precursors and thrombocytopenia) and positive response to a minimum of 5 days of antibiotic treatment. The cases defined as suspected sepsis had to have at least one symptom and one abnormal laboratory test value, and a positive response to antibiotic treatment. 
We performed a post-hoc power analysis by simulations using $\mathrm{R}$ software. All calculations were carried out using the Microsoft Statistical Package for Social Sciences (SPSS Inc., Chicago, IL, USA) for Windows v18.0. Categorical variables were compared with the chi-square test and Fisher's exact test when appropriate. Data with continuous variables were assessed with a T-test when the data followed a normal distribution and with a Mann-Whitney $U$ test if the data did not follow a normal distribution. We used univariate and multivariate logistic regression to estimate relative risks represented by odds ratios (ORs) with $95 \%$ confidence intervals (CIs). The risk factors (seen in Table 4) of caesarean section, maternal infections and neonatal infections were assessed with logistic regression. In multivariate regression analysis, the time of amniotomy after balloon expulsion and the time of oxytocin administration for IOL after amniotomy were used as continuous variables. A P-value of $<0.05$ was considered significant.

\section{Results}

The indications for IOL among 432 nulliparous women are shown in Table 1 . The mean gestational age was 41.0 $( \pm 1.4)$ at the start of IOL. The mean age of the women was $30.5( \pm 4.8)$ with mean BMI of $24.5( \pm 5.0)$. Table 2 shows the characteristics of the labour induction. Women with Bishop score $\leq 3$ at the start of IOL were more likely to need oxytocin induction than women with Bishop score $>3$ (50 \% vs. $37.9 \%, p=0.01)$.

Maternal outcomes are shown in Table 3. The overall caesarean section rate was $39.1 \%(n=169)$. The post-hoc power analysis indicated that using a two-sided alpha-

Table 1 Main indication for IOL $(n=432)$

\begin{tabular}{lcc}
\hline & $\mathrm{n}$ & Percent \\
\hline Post-term pregnancy & 266 & 61.6 \\
Pre-eclampsia/hypertension & 56 & 13 \\
Intrauterine growth restriction & 23 & 5.3 \\
Gestational diabetes & 29 & 6.7 \\
DM I & 3 & 0.6 \\
Oligohydramnion & 19 & 4.4 \\
Obstetric cholestasis & 16 & 3.7 \\
Maternal disease & 3 & 0.6 \\
Foetal disease $^{\mathrm{b}}$ & 3 & 1 \\
Psychosocial reasons $^{c}$ & 3 & 0.6 \\
Decreased foetal movement & 6 & 1.4 \\
LGA & 4 & 0.9 \\
\hline
\end{tabular}

DM I diabetes mellitus type 1, LGA large for gestational age

${ }^{a}$ Back pain, heart transplantation, difficulty urinating, ulcerative colitis

${ }^{b}$ Heart malformation, Catc-22 h, suspected distress

${ }^{c}$ Maternal exhaustion, fear of labour, maternal preference
Table $\mathbf{2}$ Characteristics of the labour induction

\begin{tabular}{lrc}
\hline & $\mathrm{n}$ & Percent \\
\hline Bishop score $\leq 3$ at start of IOL & 209 & 47.9 \\
Time the Foley catheter was retained, median (range) & 666 & $(1-1647)$ \\
Spontaneous rupture of the membranes & 25 & 5.8 \\
Spontaneous contractions before balloon expulsion & 60 & 13.9 \\
Oxytocin administration & 402 & 93.1 \\
Oxytocin needed for induction & 188 & 46.7 \\
Early epidural analgesia $^{\text {a }}$ & 15 & 3.5 \\
Primary inertia $^{\mathrm{b}}$ & 15 & 3.5 \\
\hline
\end{tabular}

${ }^{a}$ On maternal request in the absence of regular contractions

${ }^{b}$ No contractions despite oxytocin administration for $\geq 6$ hours

level of $5 \%$ we had more than $80 \%$ power to detect an odds-ratio (OR) of 1.9 between gestational diabetes $(n=81)$ and rate of cesarean delivery, and an OR of 4.6 between gestational diabetes and neonatal sepsis. Furthermore, we had over $85 \%$ power to detect an OR of 3.3 between need of oxytocin $(n=188)$ and labor induction, and an OR of 11.1 between early epidural anesthesia $(n=15)$ and labor induction.

In univariate analysis, women who underwent caesarean section were slightly older $(\geq 37$ years $)(p=0.05)$, more obese $\left(\geq 30 \mathrm{~kg} / \mathrm{m}^{2}\right)(p=0.029)$, more often had gestational diabetes $(p=0.01)$, more often had a Bishop score $\leq 3$ at start of IOL $(p=0.02)$, were more likely to need oxytocin for labour induction $(p=<0.001)$ and were more likely to have early epidural analgesia ( $p=$ 0.002) than women with vaginal delivery (Table 4). After multivariate logistic regression, only the associations with oxytocin induction and early epidural analgesia remained significant [OR 2.9 (95 \% CI 1.8-4.5); $p<0.001$ and OR 9.9 (95 \% CI 2.1-47.5); $p=0.004$ ]. Immediate or delayed timing of amniotomy or oxytocin administration were not associated with mode of delivery (Table 4). The indications for caesarean section are shown in Table 5. The median time from induction to birth was longer in women with caesarean delivery than in women with vaginal delivery (2090 min [range 170-4930] vs. $1706 \mathrm{~min}$ [range 445-4285]; $p=0.001$ ).

The maternal intrapartum infection rate was $6.3 \%$ $(n=27)$, and the postpartum infection rate was $3.9 \%$ $(n=17)$ (Table 3$)$. Both intrapartum and postpartum infections were more common among women who had caesarean sections than women who delivered vaginally [OR 7.7 (95 \% CI 2.9-20.8); $p<0.001$ and OR 3.9 (95\% CI 1.4-11.4); $p=0.007$ ]. Overall $2.8 \%(n=12)$ of the women had caesarean sections due to suspected intrapartum infection. Two of these women had blood culturepositive sepsis. In univariate analysis, gestational diabetes was associated with increased risk of intrapartum infection $(p=0.004)$ (Table 4$)$ and this association remained 
Table 3 Maternal and neonatal outcomes

\begin{tabular}{|c|c|c|c|c|c|}
\hline & \multirow{2}{*}{\multicolumn{2}{|c|}{$\begin{array}{l}\text { Caesarean delivery } \\
n=169\end{array}$}} & \multirow{2}{*}{\multicolumn{2}{|c|}{$\begin{array}{l}\text { Vaginal delivery } \\
n=263\end{array}$}} & \multirow[t]{3}{*}{ p-value } \\
\hline & & & & & \\
\hline & $\mathrm{n}$ & $\%$ & $\mathrm{n}$ & $\%$ & \\
\hline \multicolumn{6}{|l|}{ Maternal outcomes } \\
\hline Prophylactic antibiotic & 154 & 91.1 & 87 & 33.1 & $<0.001$ \\
\hline Oxytocin administration & 158 & 93.5 & 244 & 92.8 & 0.78 \\
\hline For induction of labour & 103 & 60.9 & 85 & 32.2 & $<0.001$ \\
\hline Epidural/spinal analgesia & 129 & 76.3 & 241 & 91.6 & $<0.001$ \\
\hline Received before start of regular contractions & 13 & 7.7 & 2 & 0.8 & $<0.001$ \\
\hline Foetal scalp blood sampling & 73 & 43.2 & 62 & 23.6 & $<0.001$ \\
\hline Postpartum haemorrhage $\geq 1000 \mathrm{ml}$ & 55 & 32.5 & 37 & 14.1 & $<0.001$ \\
\hline Meconium-stained amniotic fluid & 59 & 34.9 & 61 & 23.2 & 0.008 \\
\hline Maternal intrapartum infection & 22 & 13 & 5 & 1.9 & $<0.001$ \\
\hline Maternal postpartum infection & 12 & 7.1 & 5 & 1.9 & 0.007 \\
\hline Endometritis & 5 & & 4 & & \\
\hline Wound infection & 5 & & 1 & & \\
\hline Unspecified fever & 2 & & 0 & & \\
\hline \multicolumn{6}{|l|}{ Neonatal outcomes } \\
\hline Gestational weeks at delivery, mean (SD) & 41.1 & (1.3) & 41,1 & (1.4) & 0.83 \\
\hline Male & 99 & 58.6 & 127 & 48.3 & 0.04 \\
\hline Birth weight, mean(SD) & 3720 & (543) & 3530 & $(483)$ & $<0.001$ \\
\hline Macrosomia (>4500 g) & 11 & 6.5 & 3 & 1.1 & 0.004 \\
\hline Apgar $1 \min <7$ & 20 & 11.8 & 20 & 7.6 & 0.13 \\
\hline Apgar $5 \min <7^{a}$ & 11 & 6.5 & 6 & 2.3 & 0.023 \\
\hline Umbilical artery $\mathrm{pH} \leq 7.05^{\mathrm{b}}$ & 1 & 0.6 & 9 & 3.6 & 0.06 \\
\hline Umbilical artery Base excess ${ }^{b}$ & 2 & 1.3 & 7 & 2.8 & 0.30 \\
\hline Neonatal infection & 28 & 16.6 & 10 & 3.8 & 0.08 \\
\hline Suspected sepsis & 15 & 53.6 & 6 & 60 & 0.001 \\
\hline Clinical sepsis & 11 & 39.3 & 1 & 10 & $<0.001$ \\
\hline Unspecified infection & 2 & 1.2 & 3 & 30 & 0.85 \\
\hline Admission to neonatal intensive care unit & 4 & 2.4 & 5 & 1.9 & 0.74 \\
\hline Admission to neonatal ward & 43 & 25.4 & 24 & 9.1 & $<0.001$ \\
\hline
\end{tabular}

${ }^{a}$ Missing values 4

${ }^{\mathrm{b}}$ Missing values 23

after multivariate regression analysis [OR 4.3 (95\% CI 1.711.0); $p=0.002$ ].

Neonatal outcomes are shown in Table 3. The overall neonatal infection rate was $8.8 \%(n=38)$. No cases of blood culture-positive neonatal sepsis were found. Both clinical and suspected sepsis were more common in neonates delivered by caesarean section than in those delivered vaginally [OR 5.0 (95\% CI 2.4-10.6); $p<0.001$ and OR 18.2 (95 \% CI 2.3-142.6); $p=0.001$ ]. By univariate analysis, maternal age $\geq 37(p=0.01)$, gestational diabetes $(p=0.01)$ and early epidural analgesia $(p=0.029)$ were associated with neonatal clinical sepsis (Table 4). By multivariate regression analysis, the significance remained only for early epidural analgesia [OR 10.5 (95 \% CI 1.476); $p=0.02$ ].

\section{Discussion}

We found that the need for oxytocin for induction and early epidural analgesia were associated with caesarean delivery in nulliparous women undergoing IOL by Foley catheter. Gestational diabetes was associated with maternal intrapartum infection, while early epidural analgesia was associated with neonatal infection. Surprisingly, Bishop score at the start of IOL, duration of the balloon remaining in the cervical canal, timing of amniotomy or timing of oxytocin induction had no association with 
Table 4 Univariate analysis for caesarean delivery, maternal intrapartum, postpartum and neonatal infections

\begin{tabular}{|c|c|c|c|c|c|c|c|c|c|c|c|}
\hline & \multirow[b]{2}{*}{$n$} & \multicolumn{2}{|c|}{$\begin{array}{l}\text { Caesarean delivery } \\
(\mathrm{n}=169)^{\mathrm{C}}\end{array}$} & \multicolumn{2}{|c|}{$\begin{array}{l}\text { Maternal intrapartum } \\
\text { infection }(n=27)^{d} \\
\end{array}$} & \multicolumn{2}{|c|}{$\begin{array}{l}\text { Maternal postpartum } \\
\text { infection }(\mathrm{n}=17) \\
\end{array}$} & \multicolumn{2}{|c|}{$\begin{array}{l}\text { Neonatal infection } \\
(\mathrm{n}=38)\end{array}$} & \multicolumn{2}{|c|}{$\begin{array}{l}\text { Neonatal clinica } \\
\text { sepsis }(n=12)^{e}\end{array}$} \\
\hline & & OR & (CL $95 \%)$ & OR & (CL $95 \%)$ & OR & (CL $95 \%)$ & OR & (CL $95 \%)$ & OR & (CL $95 \%)$ \\
\hline Maternal age $\geq 37$ (years) & 43 & 1.9 & $1.0-3.6$ & 0.7 & $0.2-3.1$ & 1.8 & $0.2-13.9$ & 1.1 & $0.4-3.2$ & 4.9 & $1.4-17.0$ \\
\hline$I V F^{a}$ & 19 & 1.4 & $0.6-3.6$ & 0 & 0 & 0.7 & $0.1-5.8$ & 2 & $0.6-7.3$ & 0 & 0 \\
\hline Smoking & 61 & 1 & $0.6-1.8$ & 1.8 & $0.7-4.7$ & 1.2 & $0.3-5.6$ & 1.2 & $0.5-2.9$ & 2.1 & $0.5-7.9$ \\
\hline $\mathrm{BMI} \geq 30 \mathrm{~kg} / \mathrm{m}^{2}\left(\mathrm{~kg} / \mathrm{m}^{2}\right)^{\mathrm{b}}$ & 64 & 1.8 & $1.1-3.1$ & 1 & $0.3-3.0$ & 0 & 0 & 1.6 & $0.7-3.7$ & 2 & $0.5-7.5$ \\
\hline Gestational diabetes & 81 & 1.9 & $1.2-3.1$ & 3.3 & $1.5-7.4$ & 3.8 & $0.5-29.2$ & 1.4 & $0.6-3.1$ & 4.6 & $1.4-14.6$ \\
\hline Pre-eclampsia or hypertension & 68 & 1 & $0.6-1.8$ & 0.9 & $0.3-2.8$ & 0 & & 1.5 & $0.6-3.4$ & 1.5 & $0.8-9.5$ \\
\hline Bishop ${ }^{a} \leq 3$ at the start of induction & 206 & 1.6 & $1.1-2.4$ & 1.4 & $0.6-3.0$ & 1.7 & $0.6-4.7$ & 0.9 & $0.5-1.8$ & 1.5 & $0.5-4.8$ \\
\hline $\begin{array}{l}\text { Cervical effacement }>2 \mathrm{~cm} \text { at the } \\
\text { time of amniotomy }\end{array}$ & 32 & $0.8-3.4$ & $0.6-2.1$ & 1.1 & $0.2-4.7$ & 1.5 & $0.3-7.0$ & 0 & & 0 & 0 \\
\hline $\begin{array}{l}\text { Cervical dilation } \leq 3 \mathrm{~cm} \text { at the time } \\
\text { of amniotomy }\end{array}$ & 19 & 1.6 & $0.8-3.4$ & 0.7 & $0.2-3.0$ & 0.9 & $0.1-7.7$ & 0.99 & $0.3-3.0$ & 1.2 & $0.2-10.0$ \\
\hline \multicolumn{12}{|c|}{ Time of amniotomy after balloon expulsion } \\
\hline$\leq 2 \mathrm{~h}$ vs. $>2 \mathrm{~h}$ & 195 & 1.4 & $0.9-2.0$ & 0.9 & $0.4-2.0$ & 1.6 & $0.6-4.4$ & 1 & $0.5-2.1$ & 1.3 & $0.4-4.3$ \\
\hline$\leq 12 \mathrm{~h}$ vs. $>12 \mathrm{~h}$ & 47 & 0.9 & $0.5-1.7$ & 2.5 & $0.9-6.5$ & 2 & $0.3-15.6$ & 0.8 & $0.3-2.2$ & 1.7 & $0.4-8.2$ \\
\hline Oxytocin for induction of labor & 188 & 3.3 & $2.2-4.9$ & 1 & $0.5-2.3$ & 1.1 & $0.4-3.0$ & 1.2 & $0.6-2.4$ & 1.6 & $0.5-5.3$ \\
\hline \multicolumn{12}{|c|}{ Time of starting oxytocin for IOL after amniotomy } \\
\hline$\leq 3 \mathrm{~h}$ vs. $>3 \mathrm{~h}$ & 103 & 1.1 & $0.6-1.9$ & 1.8 & $0.5-5.8$ & 0.5 & $0.1-2.5$ & 0.8 & $0.3-2.4$ & 0.4 & $0.4-3.9$ \\
\hline$\leq 12 \mathrm{~h}$ vs. $>12 \mathrm{~h}$ & 52 & 1.3 & $0.7-2.5$ & 2 & $0.4-9.5$ & & & 1.8 & $0.6-5.4$ & 2.7 & $0.4-19.4$ \\
\hline Early epidural analgesia & 15 & 11.2 & $2.5-50.5$ & 1 & $0.5-2.3$ & 0.5 & $0.1-4.1$ & 2.7 & $0.7-10.0$ & 6 & $1.2-30.3$ \\
\hline
\end{tabular}

IVF; in vitro fertilization, BMI; body mass index

${ }^{\mathrm{a}}$ Missing values 2

${ }^{\mathrm{b}}$ Missing values 4

${ }^{c}$ Maternal age $\geq 37(p=0.045), B M l \geq 30(p=0.029)$, gestational diabetes $(p=0.01)$, Bishop $\leq 3(p=0.018)$, oxytocin induction ( $\left.<<0.001\right)$, early epidural analgesia ( $\left.p=0.002\right)$

${ }^{\mathrm{d}}$ Gestational diabetes $(\mathrm{p}=0.004)$

${ }^{e}$ Maternal age $\geq 37(p=0.013)$, gestational diabetes $(p=0.01)$, early epidural analgesia $(p=0.029)$

caesarean section or infections. We acknowledge the limitations of our retrospective single-institution study; we may have been susceptible to selection bias, and our results may not be applicable to other settings.

The caesarean section rate in our study was $39.1 \%$. Frederiks et al. found a similar $42 \%$ caesarean delivery rate in nulliparous women with a variety of induction methods [8]. In another recent study, the overall rate of

Table 5 Main indication for caesarean delivery $(n=169)$

\begin{tabular}{llc}
\hline & $\mathrm{n}$ & Percent \\
\hline Foetal distress & 63 & 37.3 \\
Infection & 12 & 7.1 \\
Failure to progress & 85 & 50.3 \\
$\quad$ Labour arrest & 53 & 31.4 \\
$\quad$ Failed induction & 32 & 18.9 \\
Bleeding & 1 & 0.6 \\
Pre-eclampsia & 3 & 1.8 \\
Umbilical cord prolapse & 1 & 0.6 \\
Maternal exhaustion & 2 & 1.2 \\
Failed vaginal instrumental delivery & 2 & 1.2 \\
\hline
\end{tabular}

successful IOL in combination with different methods and an unfavourable Bishop score $(<6)$ at 41 weeks of gestation was $51.3 \%$ [7]. We found a higher rate of successful labour induction resulting in vaginal delivery than these other recent studies. (The caesarean section rate among the 59 excluded women with sequential use of Foley catheter and intravaginal prostaglandin was even higher, $45.8 \%(n=27))$.

The degree of cervical ripeness in this study was assessed using the Bishop score, which was originally derived from observations made on multiparous women [12]. We used Bishop score $\geq 6$ as a marker for a ripened cervix, at which time amniotomy and oxytocin augmentation may be used. Perhaps a more modern concept for a favourable cervix in a nulliparous women would be Bishop score $\geq 8$ [13].

In previous studies, low Bishop score $(<5)$, increased maternal age, obesity and large birth weight have been associated with induction failure and operative delivery $[4,5,8,14-17]$. This trend was also seen in our study. However, in multivariate regression analysis, only the need for oxytocin to induce contractions and early epidural analgesia remained associated with caesarean delivery, as 
also seen in a previous study [18]. It has been suggested that since IOL, oxytocin use, low parity, prelabour rupture of membranes and obesity are all linked with increased caesarean delivery risk [19], this may explain the increased caesarean rate related to early epidural [20]. However, the request for analgesia early in labour (cervical dilation of $\leq$ $3 \mathrm{~cm}$ ) may be a marker for other risk factors for caesarean delivery [21].

The most common indication for caesarean delivery was failure to progress (failed induction and labour arrest), as was also noted in previous studies $[3,5]$. Several studies suggest that a substantial proportion of women undergoing IOL and remaining in the latent phase for 12-18 hours with oxytocin administration and ruptured membranes will deliver vaginally if induction is continued [22-24]. Previous studies have shown that active labour does not occur until about $6 \mathrm{~cm}$ of cervical dilation in women undergoing IOL, and that a diagnosis of failed induction should be made with caution prior to that stage $[25,26]$. ACOG clinical guidelines recommend that arrest in the first stage of labour should be reserved for women $\geq 6 \mathrm{~cm}$ of cervical dilation with ruptured membranes who are failing to progress despite of 4 hours of adequate uterine activity or 6 hours of oxytocin administration without cervical change [11]. In our study, the definitions for failed induction and arrest of labour varied. This suggests that some women undergoing IOL in our clinic might have been diagnosed with failed induction or labour arrest too early.

Macones et al. demonstrated that early amniotomy at $\leq 4 \mathrm{~cm}$ of cervical dilation during IOL shortened the time to delivery in nulliparous women, but did not impact the caesarean delivery rate [27], which was in line with our results. Oxytocin is more often used with Foley catheter IOL compared to pharmaceutical IOL or spontaneous labour [3]. Oxytocin use was high in our study, which may partly explain our high caesarean section rate. The timing of oxytocin administration for IOL was not associated with caesarean section or maternal or neonatal infections. To our knowledge, no other study has focused on this in the context of Foley catheter IOL.

In one previous study, Foley catheter IOL was associated with increased infectious morbidity [28]. In several other studies, as well as in a recent Cochrane review, Foley catheter IOL has not been linked to increased maternal or neonatal infection rates $[3,29,30]$. This is in agreement with our results. In our study, gestational diabetes was associated with maternal intrapartum infection. In a previous Danish study, type II or gestational diabetes showed a modestly increased risk of postcaesarean infection [31]. In our study, the rate of neonatal clinical sepsis was similar to that in previous studies [3, 29], but the rate of suspected neonatal infections was higher. We believe that potential bias related to
Foley catheter induction and longer induction-to-delivery interval resulted in more neonates being admitted to the neonatal ward for observation or antibiotic administration. The duration of labour after ruptured membranes has previously been linked to neonatal infections $[9,10]$. In our study, early epidural analgesia was associated with caesarean delivery and neonatal infections. This can be explained by the prolongation of labour.

\section{Conclusions}

Multivariate analysis found that early epidural analgesia and the need for oxytocin to induce contractions were associated with caesarean section in nulliparous women undergoing Foley catheter IOL. Gestational diabetes and early epidural analgesia were associated with infectious morbidity. These data are important when optimising the management of induced labour. The decision to induce labour should be carefully considered since the process has risks associated with it.

\section{Abbreviations}

IOL: Induction of labour; GBS: Streptococcus agalactie B; OR: Odds ratio; $\mathrm{Cl}$ : Confidence interval; IVF: In vitro fertilisation; BMI: Body mass index.

\section{Competing interests}

The authors explicitly state that there are no conflicts of interest in connection with this article.

\section{Authors' contributions}

This study was designed by JP, LR and HK in collaboration with OH, AAT, VMU and IN. Data analysis was performed by LR. The manuscript was drafted by $\mathrm{HK}, \mathrm{OH}$, JP and LR. All authors contributed to the final version of the manuscript. The final version of the manuscript was read and approved by all authors.

\section{Acknowledgements}

We thank Pasi Korhonen and Epid Reserch Oy for providing the manuscript statistical assistance. This study was supported by a grant from the Finnish Medical Society Duodecim, and Helsinki University Hospital Research grant (Nr. TYH2013340).

\section{Author details}

${ }^{1}$ Department of Obstetrics and Gynecology, University of Helsinki, Helsinki University Hospital, Haartmaninkatu 2, Helsinki 00029 HUS, Finland. ${ }^{2}$ Children's Hospital, University of Helsinki, Helsinki University Hospital, Helsinki, Finland.

Received: 26 March 2015 Accepted: 22 October 2015

Published online: 27 October 2015

\section{References}

1. Zeitlin J, Mohangoo AD, Delnord M, Cuttini M. EURO-PERISTAT Scientific Committee. The second European Perinatal Health Report: documenting changes over 6 years in the health of mothers and babies in Europe. J Epidemiol Community Health. 2013;67(12):983-5.

2. Martin JA, Hamilton BE, Ventura SJ, Osterman MJ, Mathews TJ. Births: final data for 2011. Natl Vital Stat Rep. 2013;62(1):1-69. 72.

3. Jozwiak M, Oude Rengerink K, Benthem M, van Beek E, Dijksterhuis MG, de Graaf IM, et al. Foley catheter versus vaginal prostaglandin E2 gel for induction of labour at term (PROBAAT trial): an open-label, randomised controlled trial. Lancet. 2011;378(9809):2095-103.

4. Beta J, Issat T, Nowicka MA, Jakimiuk AJ. Risk factors for cesarean section after using the Foley catheter for labor induction. Ginekol Pol. 2013;84(5):359-62. 
5. Vrouenraets FP, Roumen FJ, Dehing CJ, van den Akker ES, Aarts MJ, Scheve EJ. Bishop score and risk of cesarean delivery after induction of labor in nulliparous women. Obstet Gynecol. 2005;105(4):690-7.

6. Aghideh FK, Mullin PM, Ingles S, Ouzounian JG, Opper N, Wilson ML, et al. A comparison of obstetrical outcomes with labor induction agents used at term. J Matern Fetal Neonatal Med. 2014;27(6):592-6.

7. Marroquin GA, Tudorica N, Salafia CM, Hecht R, Mikhail M. Induction of labor at 41 weeks of pregnancy among primiparas with an unfavorable Bishop score. Arch Gynecol Obstet. 2013;288(5):989-93.

8. Frederiks F, Lee S, Dekker G. Risk factors for failed induction in nulliparous women. J Matern Fetal Neonatal Med. 2012;25(12):2479-87.

9. Tran $\mathrm{SH}$, Cheng YW, Kaimal AJ, Caughey AB. Length of rupture of membranes in the setting of premature rupture of membranes at term and infectious maternal morbidity. Am J Obstet Gynecol. 2008;198(6):700. e1-700.e5.

10. Herbst A, Kallen K. Time between membrane rupture and delivery and septicemia in term neonates. Obstet Gynecol. 2007;110(3):612-8.

11. American College of Obstetricians and Gynecologists (College), Society for Maternal-Fetal Medicine, Caughey AB, Cahill AG, Guise JM, Rouse DJ. Safe prevention of the primary cesarean delivery. Am J Obstet Gynecol. 2014;210(3):179-93.

12. BISHOP EH. Pelvic Scoring for Elective Induction. Obstet Gynecol. 1964;24:266-8

13. ACOG Committee on Practice Bulletins - Obstetrics. ACOG Practice Bulletin No. 107: Induction of labor. Obstet Gynecol. 2009;114(2 Pt 1):386-97.

14. O'Dwyer V, O'Kelly S, Monaghan B, Rowan A, Farah N, Turner MJ. Maternal obesity and induction of labor. Acta Obstet Gynecol Scand. 2013;92(12):1414-8.

15. Wolfe H, Timofeev J, Tefera E, Desale S, Driggers RW. Risk of cesarean in obese nulliparous women with unfavorable cervix: elective induction vs expectant management at term. Am J Obstet Gynecol. 2014;211(1):53. e1-53.e5.

16. Saylawala MA, Horton A. Effect of obesity on outcomes in nulliparous women undergoing balloon ripening for labor induction. Obstet Gynecol. 2014;123 Suppl 1:77S.

17. Fraser WD, Marcoux S, Moutquin JM, Christen A. Effect of early amniotomy on the risk of dystocia in nulliparous women. The Canadian Early Amniotomy Study Group. N Engl J Med. 1993:328(16):1145-9.

18. Lieberman E, Lang JM, Cohen A, D'Agostino Jr R, Datta S, Frigoletto Jr FD. Association of epidural analgesia with cesarean delivery in nulliparas. Obstet Gynecol. 1996;88(6):993-1000.

19. Moore AR, Shan WL, Hatzakorzian R. Predicting early epidurals: association of maternal, labor, and neonatal characteristics with epidural analgesia initiation at a cervical dilation of $3 \mathrm{~cm}$ or less. Local Reg Anesth. 2013:6:25-9.

20. Hin LY, Lau TK, Rogers M, Chang AM. Antepartum and intrapartum prediction of cesarean need: risk scoring in singleton pregnancies. Obstet Gynecol. 1997;90(2):183-6.

21. Wong CA, Scavone BM, Peaceman AM, McCarthy RJ, Sullivan JT, Diaz NT, et al. The risk of cesarean delivery with neuraxial analgesia given early versus late in labor. N Engl J Med. 2005;352(7):655-65.

22. Rouse DJ, Owen J, Hauth JC. Criteria for failed labor induction: prospective evaluation of a standardized protocol. Obstet Gynecol. 2000;96(5 Pt 1):671-7.

23. Rouse DJ, Weiner SJ, Bloom SL, Varner MW, Spong CY, Ramin SM, et al. Failed labor induction: toward an objective diagnosis. Obstet Gynecol. 2011;117(2 Pt 1):267-72.

24. Simon CE, Grobman WA. When has an induction failed? Obstet Gynecol. 2005;105(4):705-9.

25. Tuuli MG, Keegan MB, Odibo AO, Roehl K, Macones GA, Cahill AG. Progress of labor in women induced with misoprostol versus the Foley catheter. Am J Obstet Gynecol. 2013;209(3):237. e1-237.e7.

26. Harper LM, Caughey AB, Odibo AO, Roehl KA, Zhao Q, Cahill AG. Normal progress of induced labor. Obstet Gynecol. 2012;119(6):1113-8.

27. Macones GA, Cahill A, Stamilio DM, Odibo AO. The efficacy of early amniotomy in nulliparous labor induction: a randomized controlled trial. Am J Obstet Gynecol. 2012;207(5):403. e1-403.e5.

28. Heinemann J, Gillen G, Sanchez-Ramos L, Kaunitz AM. Do mechanical methods of cervical ripening increase infectious morbidity? A systematic review. Am J Obstet Gynecol. 2008;199(2):177-87. discussion 187-8.

29. Prager M, Eneroth-Grimfors E, Edlund M, Marions L. A randomised controlled trial of intravaginal dinoprostone, intravaginal misoprostol and transcervical balloon catheter for labour induction. BJOG. 2008;115(11):1443-50.
30. Jozwiak M, Bloemenkamp KW, Kelly AJ, Mol BW, Irion O, Boulvain M. Mechanical methods for induction of labour. Cochrane Database Syst Rev. 2012;3:CD001233.

31. Leth RA, Uldbjerg N, Norgaard M, Moller JK, Thomsen RW. Obesity, diabetes, and the risk of infections diagnosed in hospital and post-discharge infections after cesarean section: a prospective cohort study. Acta Obstet Gynecol Scand. 2011;90(5):501-9.

\section{Submit your next manuscript to BioMed Central and take full advantage of:}

- Convenient online submission

- Thorough peer review

- No space constraints or color figure charges

- Immediate publication on acceptance

- Inclusion in PubMed, CAS, Scopus and Google Scholar

- Research which is freely available for redistribution 